\title{
AD-HOC PROTOCOL MODELLING, VALIDATION AND AUTOMATED TEST SUITE GENERATION
}

\author{
Endre Horváth \\ Conformance Lab, Ericsson Hungary Ltd. H-1037 Budapest, Laborc u. 1, Hungary \\ Endre.Horvath@eth.ericsson.se
}

\begin{abstract}
This paper describes a trial work connected to research activity in the ad-hoc network protocols area. The goal of this work was to model and validate the Bluetooth baseband protocol layer and to generate test suite in an automated way. This was achieved by using the Telelogic TAU tools: the model was completed in SDL (using SDT), the Validator tool was used to validate it and to generate TTCN test suite.
\end{abstract}

Key words: SDL, TTCN, Bluetooth, Validation, Test suite generation

\section{INTRODUCTION}

Real-time aspects in protocol modelling, simulation and validation are very important today. Modern systems in the wireless world, like Bluetooth, have very hard time constraints, so demands on the specification languages, simulation and validation tools used in protocol technology are high. The main goal of my work was to specify a complete system to simulate and validate the Bluetooth baseband protocol layer and to generate TTCN test suite automatically, if it is possible. This paper describes how SDL was deployed in modelling, simulation and validation of Bluetooth, which workarounds were made to prepare the model for automated test suite generation.

Section 2 gives a brief introduction to Bluetooth concentrating on timing aspects. The most important modelling problems and the chosen solutions are described in Section 3, while Section 4 presents the validation procedure. 
The test suite generation work is presented in Section 5 and at the end Section 6 summarizes the experiences of my work.

\section{BLUETOOTH}

Bluetooth is a universal radio interface that enables portable electronic devices to communicate wirelessly via short-range ad-hoc networks [1]. It eliminates the need for wires, cables and connectors between cordless or mobile phones, modems, headsets, personal digital assistants, computers, printers, etc.

Each unit can communicate simultaneously with up to seven other units forming a so-called piconet (Figure 1). One Bluetooth unit acts as the master of the piconet, whereas the other unit(s) act(s) as slave(s). Each piconet can have only one master. However, slaves can participate in different piconets on a time-division multiplex basis. In addition, a master in one piconet can be a slave in other piconet(s) [1].

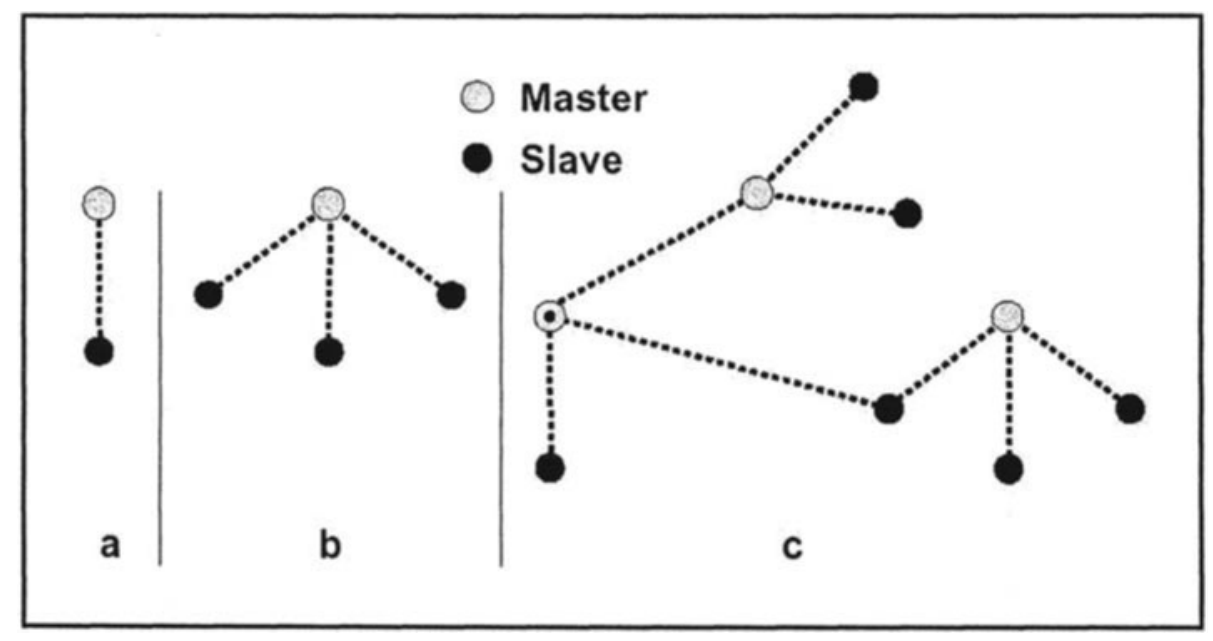

Figure 11. Bluetooth piconets [1]: a) Simple communication; b) Bluetooth piconet; c) Bluetooth scatternet

The Bluetooth radio frequency system is represented as a frequency hopping spread spectrum system in which packets are transmitted in defined time slots on defined frequencies. The channel is represented by a pseudorandom hopping sequence, hopping through the $79 \mathrm{RF}$ channels [1]. The hopping sequence is unique for the piconet and is determined by the master's Bluetooth device-address while the phase in the hopping sequence is 
determined by the master's clock. The channel is divided into time slots where each slot corresponds to an RF hop frequency. The channel hops to a new frequency in every slot, where the nominal hop rate is 1600 hops/s (slot length is $625 \mu \mathrm{s}$ ) [1]. All Bluetooth units participating in the piconet are timeand hop-synchronized to the channel. To keep this synchronization, a master is required to send packets that synchronize the slaves to the master - even if the master is not sending data. All the slaves in a piconet must be within the radio range of the master, so that every slave can synchronize to the piconet using the master's transmissions.

Every Bluetooth unit has an internal system clock that determines the timing and hopping of the transceiver. The Bluetooth clock is derived from a free running native clock that never needs to be adjusted and is never turned off. For synchronization with other units, only time offsets are used that, when added to the native clock, provide temporary Bluetooth clocks which are mutually synchronized. It should be noted that the Bluetooth clock has no relation to the time of the day; it can therefore be initialized to any value. The Bluetooth clock provides the heartbeat of the Bluetooth transceiver. Its resolution is at least half of the TX or RX slot length $(312.5 \mu \mathrm{s})$ [1].

For data transmission, a frame is segmented to baseband packets whose length can be 1-5 slots (Figure 2). The data transmission means sending the baseband packets to the destination and listening to the acknowledgement packets in the next slot. The acknowledgement packets are single slot baseband packets by default. A single-bit acknowledgement is used to indicate whether the last packet was received correctly or not.

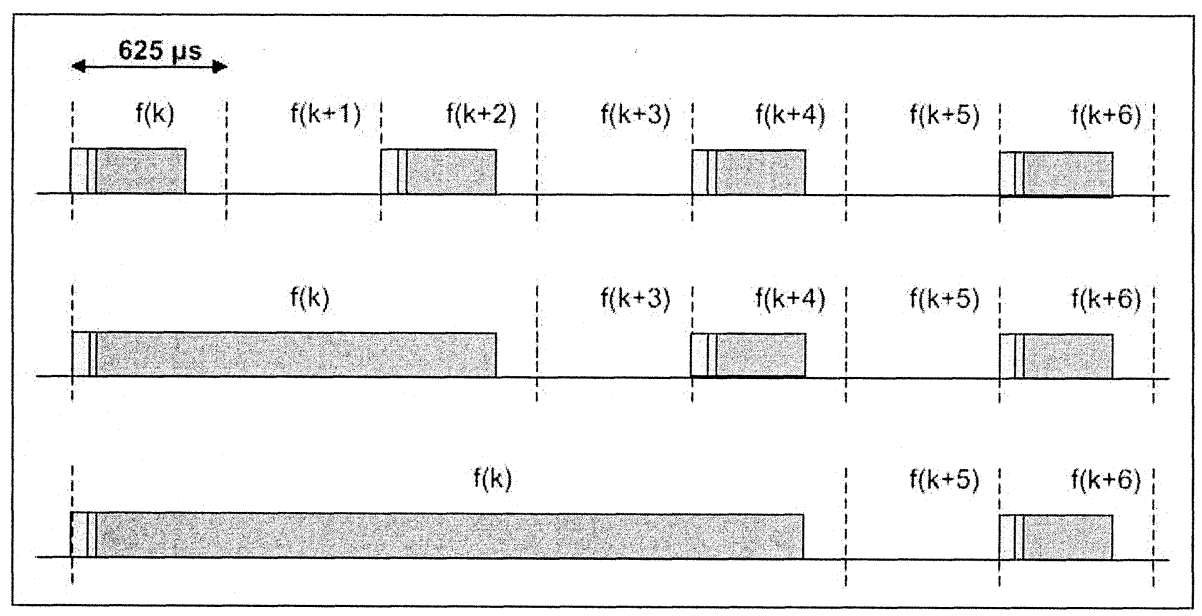

Figure 12. Baseband packets in Bluetooth [1] 


\section{SDL MODELLING OF BLUETOOTH}

Bluetooth was modelled in SDL for validation and testing purposes. The structure of the SDL system is quite simple (Figure 3): the Node block is connected to the environment via two interfaces. The task of the first one is to communicate with the upper layer (User SAP) while the other interface represents the radio channel. This structure also determines the architecture of the generated Test Suite (see Section 5).

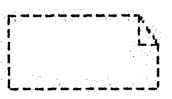

SIGNAL
Beacon(ChID, BeaconPdu), Report(ADD,APP),
Data ind(ADD,DATA), Data_reg(ADD,DATA), Data_rej,Data_res,
RTS(ChID,RTSPdu),CTSCCID,CTSPAdu),
DATA(ChID,DATAPdu),ACK(ChID,ACKPdu);

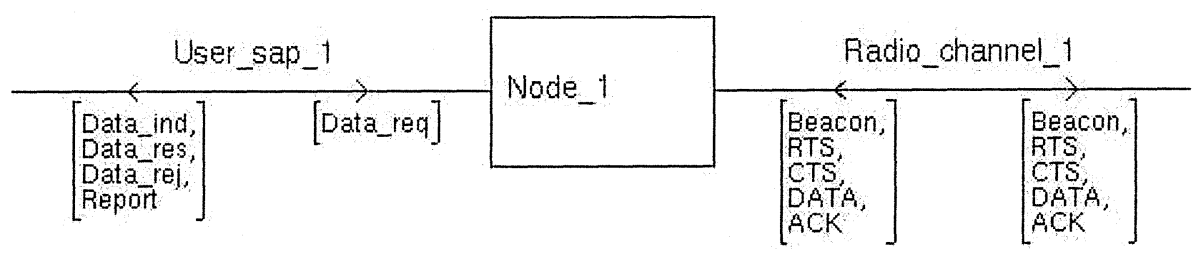

Figure 13. System structure

Unfortunately, real-time features are not fully supported in the current SDL therefore some real-time problems had to be solved by using the available language elements. For example timing in Bluetooth is essential. Every node has an internal clock that determines the timing and hopping of the transceiver. This clock is represented by a 28 bit counter where the least significant bit ticks in units of $312.5 \mu$ s (half of the slot length) [1].

An accurate cyclic timer would be nice to model Bluetooth timing in SDL, but it is not supported at the moment. First, there is no cyclic timer in SDL therefore timers have to be restarted immediately after they expired (Figure 4). If a timer expires the timeout signal gets into the input buffer of the process and there is no guarantee that the signal will be processed immediately. A solution for this problem could be to use priority inputs [2] for timeout signals (Figure 4). In this way there is a chance to avoid drifting in the Bluetooth clock. 
The 28-bit counter is implemented as a bit-string variable in the SDL model and it is increased after every timeout using a simple procedure (Figure 4). The value of the counter shows the actual status of the Bluetooth clock so it can be included in the Master's advertisement packets sent to the slaves. The actual value of this counter is the input for the frequency hop selection procedure.

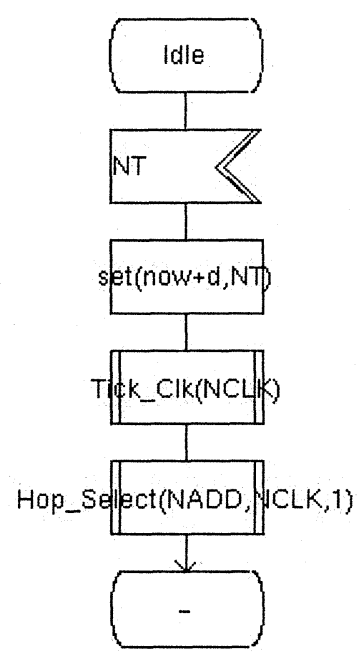

Figure 4. Using priority input for timeout signals

One of the most important tasks of the Bluetooth baseband protocol layer is to control the frequency hopping of the node's transceiver. It is realized in the protocol model by implementing the hop selection algorithm described in the Bluetooth specification [1]. This algorithm generates a pseudorandom number (a channel id) between 0 and 78 according to the MAC address and the clock status of the Master. There is also a third input parameter of the algorithm that controls how often the frequency has to be changed. In the model only two possibilities are distinguished: slow frequency hopping for scanning and fast frequency hopping for other communication. Since there is no physical layer (real transceiver) under the baseband protocol, the channel id is carried in every signal. If a signal is received the model checks whether the node is "hearing" the signal (the channel id in the signal is equal to the node's channel id) or not (Figure 5).

In Bluetooth the data frames are segmented to baseband packets of length 1-5 slots (Figure 2) [1]. There is no frequency change during the transmission of one segment, but the different segments are transmitted on different frequencies (according to the actual clock status of the piconet's master) and every segment has to be acknowledged in the next slot. This simple 
procedure is not so easy to model by using SDL, because duration cannot be assigned to sending / receiving events. The only solution is to wait until the needed slots are passed which can be achieved by using simple slot counters.

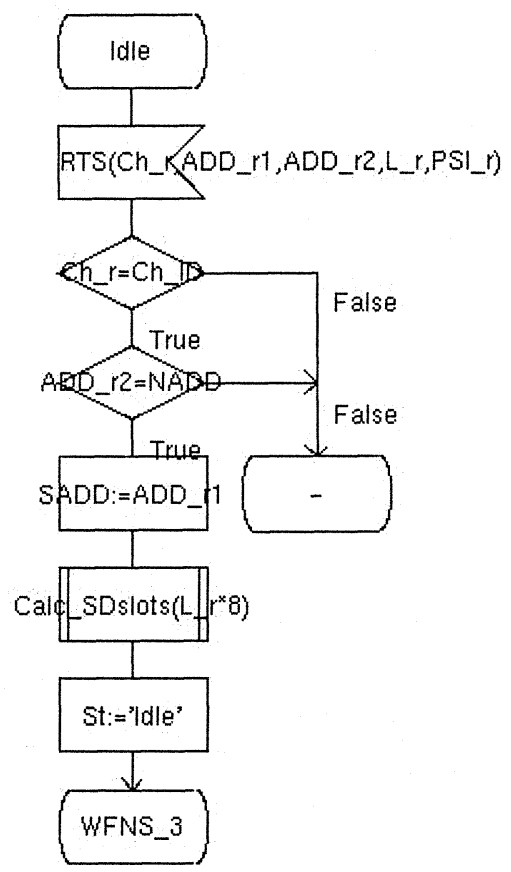

Figure 5. Checking the channel id after a signal reception

\section{VALIDATION OF THE MODEL}

The protocol model is not simple: there are many states and variables used to specify the baseband protocol of Bluetooth. Therefore the state space to be generated during validation is very large (see Figure 6), so it is not easy (or even impossible) to fully validate this SDL model. That is why the Exhaustive state space exploration algorithm was not applicable on this model and so the Bit-state algorithm was used to validate the SDL system.

Another problem is that the communication with the modelled node is possible only on specified frequencies according to the node's hop sequence. This requires well-constructed test patterns to achieve high coverage in the validation procedure. To solve this problem, the Simulator tool was used to 
produce some typical test sequences and during simulation some useful test patterns were gained that could be applied in validation, too [3].

Figure 6 shows the result of validation achieved by using simple test signals (produced during simulation) and default Validator settings (Max searching depth $=100$ ). The $72 \%$ symbol coverage could be increased up to $80 \%$ by defining some erroneous test signals (e.g. negative acknowledgement in data transmission) and by raising the searching depth. The reasons for only $80 \%$ symbol coverage are the following:

- The modelled node has a fix MAC address, while the hop selection procedure is executed according to this address. It means that some parts (e.g. permutation according to the input address bits) of this procedure could not be reached at all.

- The length of test data is limited in the Validator, therefore it was not possible to validate all the segmentation functionalities.

- Due to limitations in time and computer resources the searching depth of the Bit-state exploration algorithm was also limited and therewith increasing the searching depth raises the collision risk in application of Bit-state exploration, too.

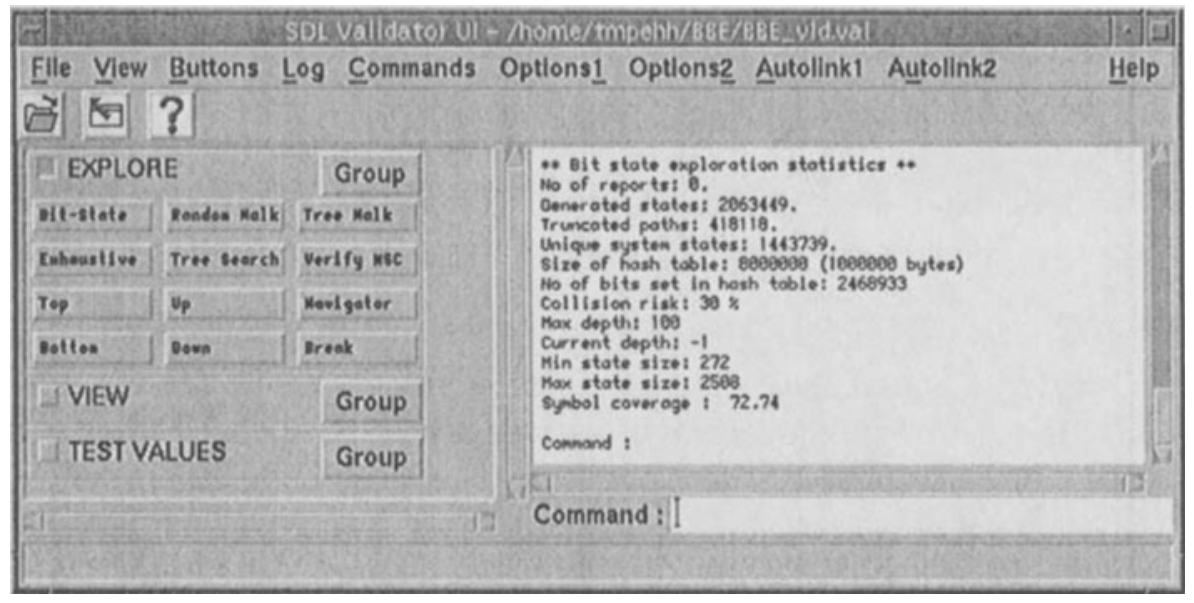

Figure 6. Validation result by using default settings

As a result it can be said that serious problem was not found during validation. The validation procedure was helpful to complete the right SDL model because some design failures were detected and only some model specific problems were occurred like exceeding the maximum transition length (reason: the hop selection algorithm is used very often). 


\section{AUTOMATED TEST SUITE GENERATION}

The Telelogic TAU tools were used for TTCN test suite generation [4]. The starting point of the generation is the SDL specification and the goal is to get a TTCN test suite in an automated way. This procedure has two main parts:

1. Producing MSC diagrams [5] that are the input test purposes for deriving TTCN test cases;

2. Generating a TTCN test suite [6] from the MSC test purposes and the SDL specification.

The second part of the generation procedure is implemented in the TAU Validator in a fully automated way [4] (on demand some translating rules can be defined to personalize the test suite) but the automation of the first part is not so trivial.

To generate MSCs automatically, an appropriate state space exploration algorithm is needed. In case of our Bluetooth model only the Bit-state algorithm could be used, because the Exhaustive algorithm was not applicable at all (see previous section), while other algorithms contain random parts that do not guarantee consistency and applicability with the MSC generation method explained in the following paragraphs. However the most important thing is not which algorithm is used in the MSC generation: the main point is the method and timing of MSC generation during the state space exploration.

A solution for these problems can be the application of an Observer process [2] (Figure 7) to generate Reports according to given rules. The Validator can store how these Reports were reached during the exploration and these paths can be saved as MSC diagrams. The outfit of the MSCs is controlled by the searching depth of the exploration algorithm and the rules defined in the Observer process. Of course the most important part of this method is the definition of the Report-generating rules. In SDL a lot of information can be collected about a process under execution, e.g. value of variables, actual state, contents of the input queue [2]. By using these information the Report generation can be controlled easily.

From a tester's perspective the model is a black box. It means that the tester sends inputs to the IUT (Implementation Under Test) and waits for the reaction. Without any reaction it is not easy to judge whether the system behaves right or not. To produce conventional test cases the outputs of the modelled system have to be observed and a Report should be generated after receiving an output. Unfortunately, observing the outputs of a process under execution is not supported in SDL explicitly, but there is a workaround to solve this problem: by a small modification to the model we can add a 
counter which value is increased after every sending event and the change of the counter's value can be watched by the observer process.
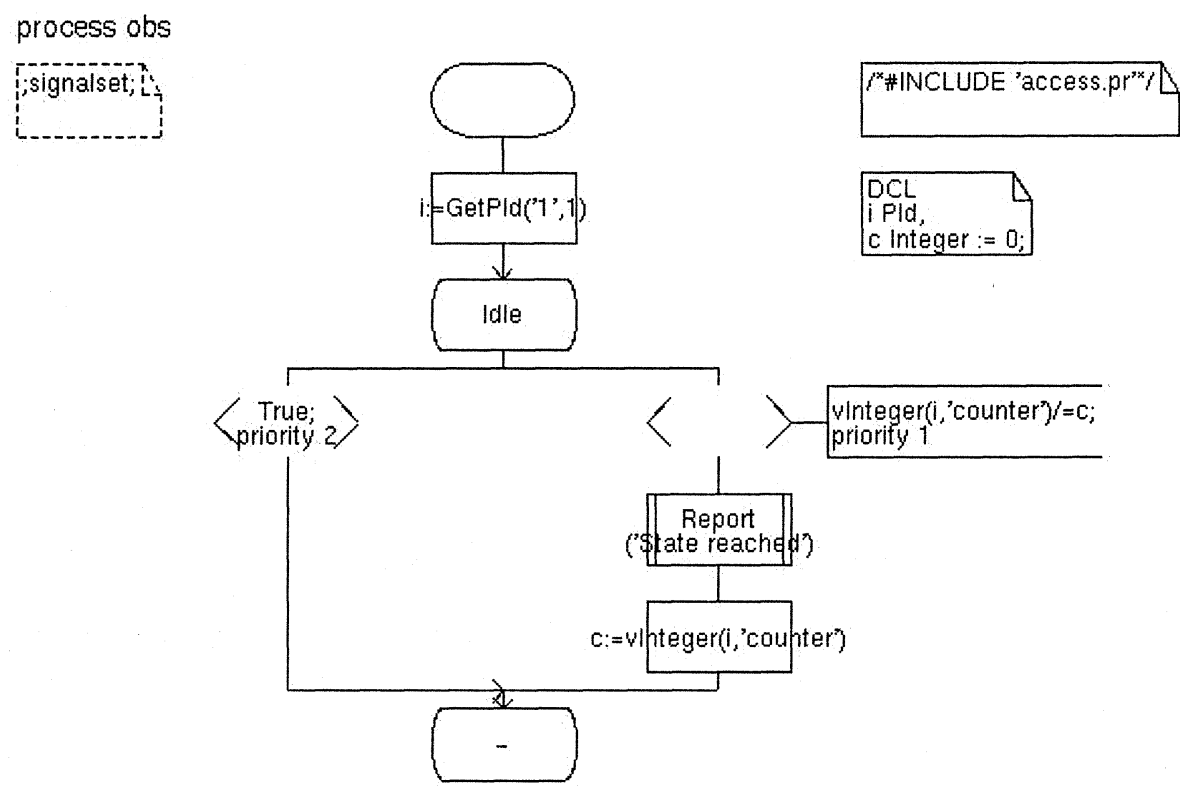

Figure 7. Observer process for MSC generation

By using this method hundreds of MSCs were generated however many of them were not significantly different from one another. The reason is that after a sending event Reports were generated also by reaching a temporal state (e.g. waiting for the next slot) and by getting into the next stable state, too. Therefore another rule was defined to reduce the number of the redundant MSCs by generating a Report only if a stable state is reached. This way 15 different MSCs were generated. An example is shown on Figure 8.

After producing the MSC test purposes the second step of the automated test suite generation was routine work. With some smart scripts and the appropriate configuration the MSCs were translated into TTCN test cases by pressing a button, so the whole test suite was generated very quickly by using the Validator tool. Figure 9 shows the TTCN test case generated from the previously presented MSC. 


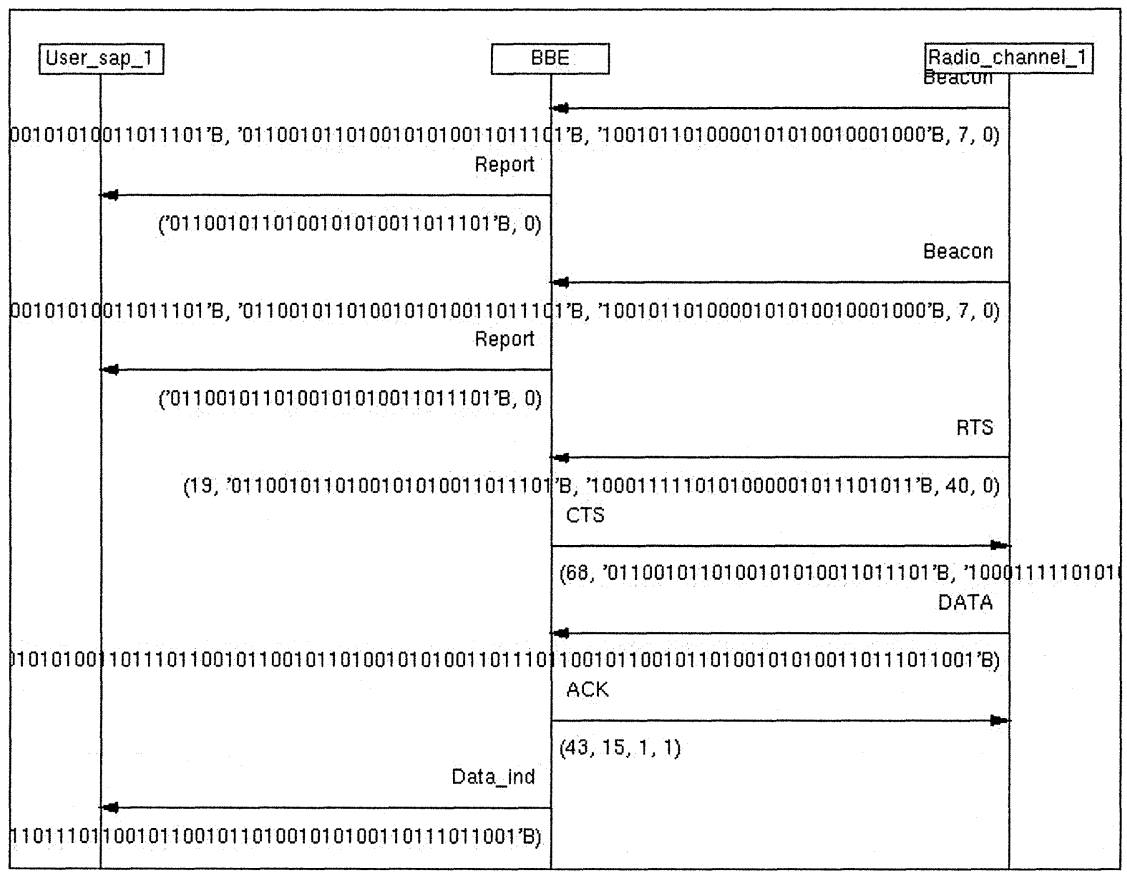

Figure 8 . Automatically generated MSC diagram

\begin{tabular}{|c|c|c|c|c|c|}
\hline \multicolumn{6}{|c|}{ Test Case Dynamic Behaviour } \\
\hline \multicolumn{2}{|c|}{$\begin{array}{l}\text { Group } \\
\text { Purpose } \\
\text { Configuration } \\
\text { Default } \\
\text { Comments } \\
\text { Selection Ref } \\
\text { Description }\end{array}$} & $\begin{array}{l}\text { me:BBE TC Asse 00007 } \\
: \\
\vdots \\
: \\
: \\
: \\
:\end{array}$ & & & \\
\hline $\mathrm{Nr}$ & Label & Behaviour Description & Constraints Ref & Verdict & Comments \\
\hline 1 & & $\begin{array}{l}\text { Radio_channel_1!Beacon } \\
\text { User sap 1? Report }\end{array}$ & $\begin{array}{l}\text { CBBE_TC_Asse_00001_004 } \\
\text { cBBE TC Asse } 00001003\end{array}$ & & \\
\hline 3 & & Radio_channel_1 I Beacon & cBBE_TC_Asse_00001_004 & & \\
\hline 4 & & User_sap_1?Report & CBBE_TC_Asse_00001_003 & & \\
\hline 5 & & Radio_channel_1 IRTS & CBBE_TC_Asse_00001_002 & & \\
\hline 6 & & Radio_channel_1? CTS & CBBE_TC_Asse_00001_001 & & \\
\hline 7 & & Radio_channel_1 IDATA & CBBE_TC_Asse_00003_003 & & \\
\hline 8 & & Radio_channel_1? ACK & CBBE_TC_Asse_00003_002 & & \\
\hline 9 & & User_sap_1? Data_ind & CBBE_TC_Asse_00003_001 & PASS & \\
\hline 10 & & User_sap_1? OTHERWISE & & FAIL & \\
\hline 11 & & Radio_channel_1? OTHERWISE & & FAlL & \\
\hline 12 & & Radio_channel_1? OTHERWISE & & FAIL & \\
\hline 13 & & Radio_channel_1? OTHERWISE & & FAIL & \\
\hline 14 & & User_sap_1? OTHERWISE & & FAIL & \\
\hline 15 & & User_sap_1? OTHERWISE & & FAIL & \\
\hline Deta & & ments: & & & \\
\hline
\end{tabular}

Figure 9. Automatically generated TTCN test case 
The quality of the generated test suite is good but the test cases have to be completed manually since there are no guard timers generated to protect the tester against deadlocks during testing (against waiting for an answer for an indefinite amount of time). However it is very positive that naming of constraints can be controlled with configuration of the generator tool and concurrent TTCN is also supported in the Validator [4].

\section{CONCLUSIONS}

The completed work described in this paper had three main phases: modelling, validation and test suite generation. The modelled protocol had some specialities that were not too easy to describe in SDL: the problem of accurate timing was solved by using priority inputs, but also the handling of frequency hopping caused some troubles. Frequency hopping was a problem in validation of the model, too. Since the default (automatically generated) test signals were not applicable in validation, the Simulator tool was used to produce the appropriate test patterns (using the right frequency identifiers). In the test suite generation phase frequency hopping was the reason again for many redundant MSCs. Even so the automated MSC generation could be controlled well by using an Observer process, and based on the generated MSCs a TTCN test suite of good quality could be generated easily.

The plan for the future is to continue this work by modelling more (up to 8) Bluetooth nodes communicating with each other. To describe this system an extra process has to be defined for modelling the radio channel. This solution makes it also possible to simulate the losing of data frames and the channel delay. The completed specification is also a very good base for experimenting with real-time extensions to SDL in terms of a European research project called Interval [7].

\section{REFERENCES}

[1] Specification of the Bluetooth System (Core), Specification Volume 1, 2001

[2] ITU-T Recommendation Z.100 - Specification and Description Language, 1996

[3] R. L. Probert, A. W. Williams: Fast Functional Test Generation Using an SDL Model, Testing of Communicating Systems, Budapest, Hungary, 1999

[4] M. Schmitt, A. Ek, B. Koch, J. Grabowski, D. Hogrefe: Autolink - Putting SDL-based test generation into practice, Testing of Communicating Systems, Tomsk, Russia, 1998

[5] ITU-T Recommendation Z.120 - Message Sequence Charts, 1996

[6] OSI - Open System Interconnection, Conformance testing methodology and framework - Part 3: Tree and Tabular Combined Notation, ISO/IEC 9646-3, 1997

[7] Preliminary Specification of Timed Extensions; Formal Design, Validation and Testing of Real-Time Telecommunications Systems; Interval project report, 2000 